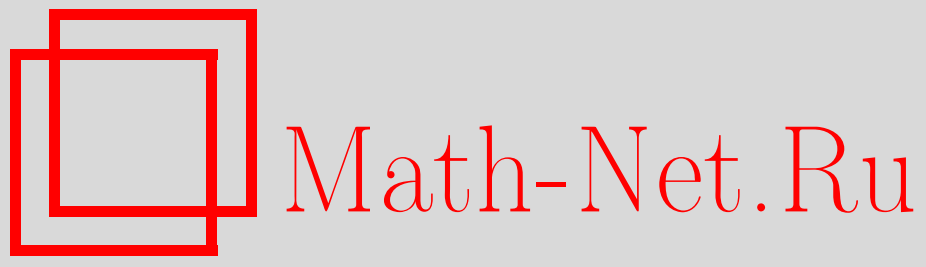

М. О. Авдеева, О статистиках неполных частных конечных цепных дробей, Функи. анализ и его прил., 2004, том 38, выпуск 2, 1-11

DOI: https://doi.org/10.4213/faa103

Использование Общероссийского математического портала MathNet.Ru подразумевает, что вы прочитали и согласны с пользовательским соглашением

http://www . mathnet.ru/rus/agreement

Параметры загрузки:

IP : 18.209 .158 .208

26 апреля 2023 г., 13:01:36

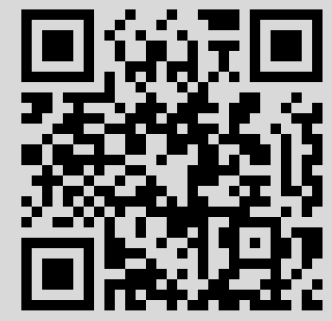


Функииональный анализ и его приложения

2004, т. 38 , вып. 2, с. 1-11

УДК $511.36+511.37$

\title{
О статистиках неполных частных конечных цепных дробей*
}

\author{
(C) 2004. М. О. АвдЕевА
}

\section{Введение}

В брошюре В. И. Арнольда [1] (см. также [2]) на с. 17 поставлена следующая задача:

«I. Рассмотрим все целые точки $(p, q)$ в положительной четверти круга радиуса $N$, т. е. такие, что $p^{2}+q^{2} \leqslant N^{2}, p>0, q>0$. Каждое рациональное число $\alpha=p / q$ разложим в цепную дробь (все эти дроби конечны). Посмотрим, сколько единиц, двоек, троек и т. д. среди элементов всех этих дробей, и определим частоты, которые будут зависеть от $N$. Пусть теперь $N$ очень велико. Будут ли эти числа близки к гауссовым вероятностям из формулы

$$
p_{k}=\frac{1}{\ln 2} \ln \left(1+\frac{1}{k(k+2)}\right) ? »
$$

Она тесно связана с метрической теорией диофантовых приближений, теорией динамических систем, а также с многими другими разделами математики.

Разложение рационального числа $r$ в конечную цепную дробь длины $s=s(r)$ с целой частью $t_{0}=[r]$ и неполными частными $t_{1}, \ldots, t_{s}$ (натуральные числа), $t_{s} \geqslant 2$, формально записывают в виде

$$
r=\left[t_{0}, t_{1}, \ldots, t_{s}\right] .
$$

Обозначим через $H_{k}(N)$ общее количество неполных частных $t_{i}$ для всех $i$, $1 \leqslant i \leqslant s(r)$, совпадающих с натуральным числом $k$, для всех дробей $r=\alpha$ из сформулированной задачи. Тогда величина

$$
H(N)=\sum_{k=1}^{\infty} H_{k}(N)
$$

есть сумма длин цепных дробей указанных выше рациональных чисел $r$.

В настоящей работе доказывается, что при растущем $N$ равномерно по $k$

$$
\frac{H_{k}(N)}{H(N)}=p_{k}+O\left(\frac{1}{\ln N}\right) .
$$

Тем самым уточняется ответ на вопрос В. И. Арнольда, содержащийся в работе [3] с более слабой оценкой $O\left((\ln N)^{-1 / 2}\right)$ остаточного члена.

Положим по определению

$$
A_{i} / B_{i}=\left[t_{i}, \ldots, t_{s}\right] \quad(1 \leqslant i \leqslant s),
$$

*Работа выполнена при финансовой поддержке Российского фонда фундаментальных исследований (грант №04-01-97000). 
где $A_{i}$ целое, а $B_{i}$ натуральное, причем НОД $\left(A_{i}, B_{i}\right)=1$. Обозначим через $s_{x}(r)$ количество индексов $i$ из разложения (0.1), для которых $B_{i} \leqslant x A_{i}$. Поскольку всегда $0<B_{i} \leqslant A_{i}$, то можно считать, что $x \in(0,1]$. Из $(0.3)$ непосредственно следует, что разность $(k=1,2,3, \ldots)$

$$
s^{(k)}(r)=s_{1 / k}(r)-s_{1 /(k+1)}(r)
$$

есть количество неполных частных $t_{i}$ в разложении $(0.1)$, совпадающих с $k$, и при этом

$$
s(r)=s_{1}(r)=\sum_{k=1}^{\infty} s^{(k)}(r) .
$$

Для положительного параметра $R \rightarrow \infty$ положим

$$
N_{x}(R)=\sum s_{x}(a / d)
$$

где суммирование проводится по всем натуральным $a$ и $d$, таким, что $a^{2}+$ $d^{2} \leqslant R^{2}$. Тогда, заменив употреблявшееся в начале статьи обозначение $N$ на $R$, получаем

$$
H(R)=N_{1}(R), \quad H_{k}(R)=N_{1 / k}(R)-N_{1 /(k+1)}(R) .
$$

Асимптотическая формула (0.2) непосредственно вытекает из следующего главного результата работы.

Tеорема. Равномерно по $x \in(0,1]$

$$
N_{x}(R)=\frac{3}{\pi} \ln (1+x) R^{2} \ln R+O\left(R^{2}\right) .
$$

Автор благодарит В. А. Быковского за полезные советы и внимание.

\section{§1. Соответствие Хейльбронна}

Напомним, что для $r$ с разложением (0.1)

$$
P_{i} / Q_{i}=\left[t_{0}, t_{1}, \ldots, t_{i}\right]
$$

есть $i$-я подходящая дробь. При этом предполагается, что $P_{i}$ - целое, а $Q_{i}-$ натуральное числа с НОД $\left(P_{i}, Q_{i}\right)=1$. Особо отметим, что разложение числа $P_{i} / Q_{i}$ в цепную дробь имеет канонический вид

(a) $(1.1)$, если $t_{i} \geqslant 2$;

(b) $P_{i} / Q_{i}=\left[t_{0}, t_{1}, \ldots, t_{i-1}+1\right]$, если $t_{i}=1$.

Нам понадобятся следующие хорошо известные соотношения (см. [4]):

$$
Q_{i} / Q_{i-1}=\left[t_{i}, t_{i-1}, \ldots, t_{1}\right], \quad P_{i-1} Q_{i}-P_{i} Q_{i-1}=(-1)^{i} .
$$

Пусть $d$ - натуральное число. Обозначим через $\Omega(d)$ множество всех $d$-четверок, т. е. четверок $\omega=\left(m, m^{\prime}, n, n^{\prime}\right)$ натуральных чисел, для которых

$$
m n^{\prime}+m^{\prime} n=d, \quad 1 \leqslant m^{\prime}<m, \quad 1 \leqslant n<n^{\prime}, \quad \text { НОД }\left(m, m^{\prime}\right)=\operatorname{HОД}\left(n, n^{\prime}\right)=1 .
$$

Хейльбронн [5] построил биективное отображение множества $\Omega(d)$ на множество

$$
\{(a ; i) \mid 1 \leqslant a \leqslant d / 2, \text { НОД }(a, d)=1,1 \leqslant i<s(a / d)\}
$$

по правилу

$$
\omega \rightarrow(a(\omega) ; i)
$$


где $a=a(\omega)$ и индекс $i$ определяются из разложений

$$
\begin{gathered}
n^{\prime} / n=\left[t_{i}, \ldots, t_{1}\right], \quad m / m^{\prime}=\left[t_{i+1}, \ldots, t_{s}\right], \\
d / a=\left[t_{1}, \ldots, t_{s}\right] .
\end{gathered}
$$

Для каждого $\omega \in \Omega(d)$ определим целое $\bar{n}=\bar{n}(\omega)$ условиями

$$
\bar{n} n \equiv 1 \quad\left(\bmod n^{\prime}\right), \quad-n^{\prime} / 2<\bar{n} \leqslant n^{\prime} / 2 .
$$

В заключение этого параграфа отметим, что множество $\Omega(d)$ является пустым только для $d=1,2,3,4,6$. При этом для остальных $d$ и любой четверки $\omega \in \Omega(d)$

$$
1<a(\omega)<d / 2
$$

\section{§2. Вспомогательные леммы}

Пусть $\theta \in(0,1 / 2]$ и

$$
\begin{gathered}
\Omega_{\theta}(d)=\{\omega \in \Omega(d) \mid a(\omega) \leqslant \theta d\} \\
\widetilde{\Omega}_{\theta}(d)=\left\{\omega \in \Omega(d) \mid-\theta n^{\prime}(\omega)<\bar{n}(\omega) \leqslant \theta n^{\prime}(\omega)\right\} .
\end{gathered}
$$

Множества $\Omega_{\theta}(d)$ и $\widetilde{\Omega}_{\theta}(d)$, вообще говоря, не совпадают. Но отличаются они друг от друга «не очень сильно». Последнее обстоятельство лежит в основе доказательства следующего утверждения, играющего в дальнейшем важную роль. Пусть \# $M$ означает число элементов множества $M$.

Лемма 1. Равномерно по $\theta \in(0,1 / 2]$

$$
\# \Omega_{\theta}(d)=\# \widetilde{\Omega}_{\theta}(d)+O(d) .
$$

ДокАЗАТЕЛЬСтво. Пусть в (1.2)

$$
Q_{i}=n^{\prime}, \quad Q_{i-1}=n, \quad Q_{i} / P_{i}=\left[t_{1}, \ldots, t_{i}\right] .
$$

Тогда из второго равенства в (1.2) следует, что

$$
(-1)^{i-1} P_{i} n \equiv 1 \quad\left(\bmod n^{\prime}\right) \text {. }
$$

Поскольку $t_{1} \geqslant 2$, то $-n^{\prime} / 2<(-1)^{i-1} P_{i} \leqslant n^{\prime} / 2$ и $\bar{n}=(-1)^{i-1} P_{i}$. Значит, $n^{\prime} /|\bar{n}|=$ $\left[t_{1}, \ldots, t_{i}\right]$. Отсюда получаем, что $|\bar{n}| / n^{\prime}$ есть подходящая дробь дроби $a / d=$ $\left[0, t_{1}, \ldots, t_{s}\right]$ и при этом (см. [4])

$$
\left|\frac{a}{d} n^{\prime}-\right| \bar{n}||<\frac{1}{n^{\prime}} .
$$

Принимая во внимание ограничения на $\omega\left(a(\omega) \leqslant \theta d\right.$ для $\Omega_{\theta}(d)$ и $-\theta n^{\prime}(\omega)<$ $\bar{n}(\omega) \leqslant \theta n^{\prime}(\omega)$ для $\left.\widetilde{\Omega}_{\theta}(d)\right)$, отсюда выводим, что общее количество $d$-четверок из теоретико-множественной симметрической разности

$$
\left(\Omega_{\theta}(d) \cup \widetilde{\Omega}_{\theta}(d)\right) \backslash\left(\Omega_{\theta}(d) \cap \widetilde{\Omega}_{\theta}(d)\right)
$$

не превосходит числа элементов множества

$$
\left\{\omega \in \Omega(d)|| \bar{n}(\omega)-\theta n^{\prime}(\omega) \mid<\frac{1}{n^{\prime}(\omega)} \text { или }\left|\bar{n}(\omega)+\theta n^{\prime}(\omega)\right|<\frac{1}{n^{\prime}(\omega)}\right\} .
$$


Для взаимно простых натуральных чисел $n$ и $n^{\prime}$ все решения уравнения $m n^{\prime}+$ $m^{\prime} n=d$ в целых $\left(m, m^{\prime}\right)$ записываются в виде

$$
m=m_{0}+n t, \quad m^{\prime}=m_{0}^{\prime}-n^{\prime} t \quad(t \in \mathbb{Z}),
$$

где $\left(m_{0}, m_{0}^{\prime}\right)$ - некоторое фиксированное решение. Условие $0<m^{\prime}<m$ эквивалентно двойному неравенству

$$
\frac{m_{0}^{\prime}-m_{0}}{n^{\prime}+n}<t<\frac{m_{0}^{\prime}}{n^{\prime}} .
$$

Поскольку

$$
\frac{m_{0}^{\prime}}{n^{\prime}}-\frac{m_{0}^{\prime}-m_{0}}{n^{\prime}+n}=\frac{d}{n^{\prime}\left(n^{\prime}+n\right)},
$$

количество элементов множества (2.1) не превосходит

$$
\sum_{1<n<n^{\prime}<d}^{( \pm)}\left(1+\frac{d}{n^{\prime}\left(n^{\prime}+n\right)}\right)
$$

где $( \pm)$ означает, что в суммировании участвуют только те пары $\left(n, n^{\prime}\right)$, для которых

$$
\left|\bar{n}-\theta n^{\prime}\right|<\frac{1}{n^{\prime}} \quad \text { или } \quad\left|\bar{n}+\theta n^{\prime}\right|<\frac{1}{n^{\prime}} .
$$

Так как $n^{\prime} \geqslant 2$, то из этих неравенств следует, что

$$
\left|\bar{n}-\theta n^{\prime}\right|<\frac{1}{2} \text { или }\left|\bar{n}+\theta n^{\prime}\right|<\frac{1}{2} .
$$

Каждое из них определяет $\bar{n}$ (а вместе с ним и $n$ ) по $n^{\prime}$ не более чем одним способом. Поэтому интересующая нас величина оценивается суммой

$$
\sum_{1<n^{\prime}<d} 2\left(1+\frac{d}{\left(n^{\prime}\right)^{2}}\right)=O(d) .
$$

Следовательно, множества $\Omega_{\theta}(d)$ и $\widetilde{\Omega}_{\theta}(d)$ отличаются друг от друга на $O(d)$ элементов. Лемма 1 доказана.

Обозначим через $\Phi_{x}(d ; P)$ количество всех пар $(\omega ; \bar{n})$ с $\omega \in \Omega(d)$ и $\bar{n} \in \mathbb{Z}$, для которых

$$
\begin{gathered}
m^{\prime}(\omega) \leqslant x m(\omega), \\
-\frac{P}{d} n^{\prime}(\omega)<\bar{n} \leqslant \frac{P}{d} n^{\prime}(\omega), \\
\bar{n} \cdot n(\omega) \equiv 1 \quad\left(\bmod n^{\prime}(\omega)\right) .
\end{gathered}
$$

Особо подчеркнем, что в данной ситуации $\bar{n}$ обозначает самостоятельную целочисленную переменную.

Лемма 2. Равномерно по $x \in(0,1]$ при $P \rightarrow \infty$

$$
\sum_{1 \leqslant a \leqslant P}^{\text {НОД }(a, d)=1} s_{x}(a / d)=\Phi_{x}(d ; P)+O(P+d) .
$$


ДокАЗАТЕЛЬСтво. Сначала рассмотрим случай $0<P \leqslant d / 2$. Заметим, что для $1 \leqslant i<s(a / d)$ пара $\left(A_{i+1}, B_{i+1}\right)$ (см. введение) совпадает с $\left(m, m^{\prime}\right)$ (см. второе разложение в (1.4)). Воспользовавшись соответствием Хейльбронна (1.3), получим равенство

$$
\sum_{1 \leqslant a \leqslant P}^{\text {НОД }(a, d)=1} s_{x}\left(\frac{a}{d}\right)=\#\left\{\omega \in \Omega_{P / d}(d) \mid m^{\prime}(\omega) \leqslant x m(\omega)\right\}+O(d) .
$$

Остаточный член оценивает количество пар $\left(A_{1}, B_{1}\right)$, которые не принимаются во внимание. Воспользовавшись леммой 1 , отсюда получаем утверждение леммы 2 в рассматриваемом случае.

Легко заметить, что для $1 \leqslant a \leqslant d / 2$ с НОД $(a, d)=1$

$$
\frac{d}{a}=\left[t_{1}, t_{2}, \ldots, t_{s}\right], \quad \frac{d}{d-a}=\left[1, t_{1}-1, t_{2}, \ldots, t_{s}\right] .
$$

Кроме того, для любого целого $k$

$$
s_{x}\left(k+\frac{a}{d}\right)=s_{x}\left(\frac{a}{d}\right) .
$$

Опираясь на эти наблюдения и периодичность по $\bmod n^{\prime}(\omega)$ решений $\bar{n}$ сравнения (2.4), распространяем утверждение леммы 2 на остальные $P>d / 2$.

\section{§3. Применение оценок сумм Клостермана}

Для натурального числа $q$ и целого $l$ положим

$$
\delta_{q}(l)=\left\{\begin{array}{lll}
1, & \text { если } l \equiv 0 & (\bmod q), \\
0, & \text { если } l \neq \equiv 0 & (\bmod q) .
\end{array}\right.
$$

Пусть $Q$ - любое целое, а натуральное число $P$ принимает значения от 1 до $q$ включительно. Для целого $w \in(Q, Q+P]$ положим

$$
\Delta_{q}(w ; P, Q)= \begin{cases}1, & \text { если } Q<w \leqslant Q+P, \\ 0, & \text { если } Q+P<w \leqslant Q+q .\end{cases}
$$

Речь идет о характеристической функции, выделяющей целые, лежащие в полуинтервале $(Q, Q+P]$, из целых, лежащих в $(Q, Q+q]$. Воспользовавшись дискретным преобразованием Фурье по модулю $q$, получим разложение

$$
\Delta_{q}(w ; P, Q)=\sum_{-q / 2<k \leqslant q / 2} \widehat{\Delta}_{q}(k ; P, Q) \exp \left(2 \pi i \frac{k w}{q}\right)
$$

с коэффициентами

$$
\widehat{\Delta}_{q}(k ; P, Q)=\frac{1}{q} \sum_{l=Q+1}^{Q+P} \exp \left(-2 \pi i \frac{k l}{q}\right) .
$$

Если $k \equiv 0(\bmod q)$, то

$$
\widehat{\Delta}_{q}(k ; P, Q)=\widehat{\Delta}_{q}(0 ; P, Q)=\frac{P}{q} .
$$


Суммируя геометрическую прогрессию, для $k \not \equiv 0(\bmod q)$ получаем

$$
\widehat{\Delta}_{q}(k ; P, Q)=\frac{1}{q} \frac{1-\exp (-2 \pi i k P / q)}{1-\exp (-2 \pi i k / q)} \exp (-2 \pi i k(Q+1) / q) .
$$

Из (3.2) и (3.3) немедленно следует хорошо известное неравенство

$$
\left|\widehat{\Delta}_{q}(k ; P, Q)\right| \leqslant \begin{cases}1, & \text { если } k=0, \\ 1 /|k|, & \text { если } 0<|k| \leqslant q / 2 .\end{cases}
$$

По определению для целых $m$ и $n$

$$
S_{q}(m, n)=\sum_{u, v=1}^{q} \delta_{q}(u v-1) \exp \left(2 \pi i \frac{m u+n v}{q}\right)
$$

есть сумма Клостермана по модулю $q$ с оценкой (для любого $\varepsilon>0$ )

$$
S_{q}(m, n) \underset{\varepsilon}{\ll} q^{1 / 2+\varepsilon} \sqrt{\mathrm{HОД}(m, q)}
$$

(см. [6]). Так как $S_{q}(m, n)=S_{q}(n, m)$, то в правой части формулы (3.5) вместо $m$ можно поставить $n$. В случае $m \equiv n \equiv 0(\bmod q)$

$$
S_{q}(m, n)=S_{q}(0,0)=\varphi(q),
$$

где $\varphi(q)$ - функция Эйлера, т.е. количество целых $k$ из $(0, q]$, для которых НОД $(k, q)=1$.

Лемма 3. Пусть $\varepsilon-$ любое положительное число. Тогда для любых $Q_{1}, Q_{2}$, $P_{1}, P_{2} \in \mathbb{R}$, таких, что $0<P_{1}, P_{2} \leqslant q$,

$$
\sum_{\substack{Q_{1}<u \leqslant Q_{1}+P_{1} \\ Q_{2}<v \leqslant Q_{2}+P_{2}}} \delta_{q}(u v-1)=\varphi(q) \frac{P_{1} P_{2}}{q^{2}}+O_{\varepsilon}\left(q^{1 / 2+\varepsilon}\right) .
$$

ДокАЗАТЕЛЬСтво. Нетрудно заметить, что достаточно рассмотреть случай с целыми $Q_{1}, Q_{2}$ и натуральными $P_{1}, P_{2}$. С помощью разложения (3.1), обозначив интересующую нас сумму через $\mathscr{D}_{q}\left(P_{1}, P_{2} ; Q_{1}, Q_{2}\right)$, получим

$$
\begin{aligned}
\mathscr{D}_{q}\left(P_{1}, P_{2} ; Q_{1}, Q_{2}\right) & =\sum_{u=Q_{1}+1}^{Q_{1}+q} \sum_{v=Q_{2}+1}^{Q_{2}+q} \delta_{q}(u v-1) \Delta_{q}\left(u ; P_{1}, Q_{1}\right) \Delta_{q}\left(v ; P_{2}, Q_{2}\right) \\
& =\sum_{-q / 2<m, n \leqslant q / 2} \widehat{\Delta}_{q}\left(m ; P_{1}, Q_{1}\right) \widehat{\Delta}_{q}\left(n ; P_{2}, Q_{2}\right) S_{q}(m, n) .
\end{aligned}
$$

Выделив из последней суммы слагаемое для $m=n=0$, с помощью соотношений (3.1)-(3.6) находим:

$$
\begin{aligned}
\mathscr{D}_{q}\left(P_{1}, P_{2} ; Q_{1}, Q_{2}\right)-\varphi(q) \frac{P_{1} P_{2}}{q^{2}} \underset{\varepsilon}{\ll} \sum_{m, n=1}^{q} \frac{1}{m n} q^{1 / 2+\varepsilon / 2} \sqrt{\text { НОД }(m, q)} \\
\ll q^{1 / 2+\varepsilon / 2} \ln (1+q) \cdot \sum_{t \backslash q} \sqrt{t} \sum_{1 \leqslant m^{\prime} t \leqslant q} \frac{1}{m^{\prime} t} \ll q^{1 / 2+\varepsilon / 2} \ln ^{2}(1+q) \sum_{t \backslash q} \frac{1}{\sqrt{t}} .
\end{aligned}
$$


Воспользовавшись стандартными оценками

$$
\ln ^{2}(1+q) \underset{\varepsilon}{\ll} q^{\varepsilon / 4}, \quad \sum_{t \backslash q} \frac{1}{\sqrt{t}} \underset{\varepsilon}{\ll} q^{\varepsilon / 4}
$$

(по поводу второй см. [7]), получим утверждение леммы 3.

ЗАмЕчАниЕ. Если в лемме 3 считать $P_{2}$ любым положительным числом, то, разбивая $\left(Q_{2}, Q_{2}+P_{2}\right)$ на полуинтервалы длины $\leqslant q$, получим асимптотическую формулу с остаточным членом

$$
O_{\varepsilon}\left(q^{1 / 2+\varepsilon}+q^{-1 / 2+\varepsilon} P_{2}\right) .
$$

Лемма 4. Пусть $q>1$. Тогда для любого положителъного $P \rightarrow \infty$ равномерно по $x \in(0,1]$ (для любого $\varepsilon>0)$

$$
\sum_{n=1}^{q-1}\left(\sum_{|\bar{n}| \leqslant P} \delta_{q}(n \bar{n}-1)\right) \frac{1}{q+x n}=2 \frac{\ln (1+x)}{x} \frac{\varphi(q)}{q^{2}} P+O_{\varepsilon}\left(q^{-1 / 2+\varepsilon}+P q^{-3 / 2+\varepsilon}\right) .
$$

ДокАзАТЕЛьство. Воспользовавшись преобразованием Абеля

$$
\sum_{1 \leqslant n \leqslant N} a(n) b(n)=\left(\sum_{1 \leqslant k \leqslant N} a(k)\right) b(N)+\sum_{1 \leqslant n<N}\left(\sum_{1 \leqslant k \leqslant n} a(k)\right)(b(n)-b(n+1))
$$

и принимая во внимание замечание к лемме 3, для рассматриваемой суммы получим выражение

$$
\begin{aligned}
& \left(\sum_{\substack{1 \leqslant u<q \\
|v| \leqslant P}} \delta_{q}(u v-1)\right) \frac{1}{q+x(q-1)} \\
& \quad+\sum_{1 \leqslant n<q-1}\left(\sum_{\substack{1 \leqslant u<n \\
|v| \leqslant P}} \delta_{q}(u v-1)\right)\left(\frac{1}{q+x n}-\frac{1}{q+x(n+1)}\right) \\
& =\left(\varphi(q) \frac{2 P}{q}+O_{\varepsilon}\left(q^{1 / 2+\varepsilon}+P q^{-1 / 2+\varepsilon}\right)\right) \frac{1}{q+x(q-1)} \\
& \quad+\sum_{1 \leqslant n<q-1}\left(\varphi(q) \frac{2 P n}{q^{2}}+O_{\varepsilon}\left(q^{1 / 2+\varepsilon}+P q^{-1 / 2+\varepsilon}\right)\right)\left(\frac{1}{q+x n}-\frac{1}{q+x(n+1)}\right) \\
& =\frac{2 \varphi(q)}{q^{2}} P \sum_{n=1}^{q-1} \frac{1}{q+x n}+O_{\varepsilon}\left(q^{-1 / 2+\varepsilon}+P q^{-3 / 2+\varepsilon}\right) .
\end{aligned}
$$

Осталось только заметить, что

$$
\sum_{n=1}^{q-1} \frac{1}{q+x n}=\int_{0}^{q} \frac{d \alpha}{q+x \alpha}+O\left(\frac{1}{q}\right)=\frac{\ln (1+x)}{x}+O\left(\frac{1}{q}\right) .
$$

Лемма 4 доказана. 
Лемма 5. В предположениях леммы 4 равномерно по $x \in(0,1]$

$$
\sum_{\substack{1 \leqslant n<q \\|\bar{n}| \leqslant P}} \frac{\delta_{q}(n \bar{n}-1)}{(q+x n)\left(q^{2}+\bar{n}^{2}\right)}=2 \frac{\ln (1+x)}{x} \frac{\varphi(q)}{q^{3}} \operatorname{arctg} \frac{P}{q}+O_{\varepsilon}\left(q^{-5 / 2+\varepsilon}\right) .
$$

ДокАЗАтЕльство. Достаточно доказать утверждение леммы для натуральных $P$. Записав нужную сумму в виде

$$
\sum_{|\bar{n}| \leqslant P} \frac{1}{q^{2}+\bar{n}^{2}}\left(\sum_{1 \leqslant n<q} \frac{\delta_{q}(n \bar{n}-1)}{q+x n}\right)
$$

и выполнив преобразование Абеля по $|\bar{n}|$, с помощью леммы 4 получим выражение

$$
\begin{aligned}
\left(\sum_{|\bar{n}| \leqslant P}\left(\sum_{1 \leqslant n<q} \frac{\delta_{q}(n \bar{n}-1)}{q+x n}\right)\right) \frac{1}{q^{2}+P^{2}} & \\
& +\sum_{N=1}^{P-1}\left(\sum_{|\bar{n}| \leqslant N}\left(\sum_{1 \leqslant n<q} \frac{\delta_{q}(n \bar{n}-1)}{q+x n}\right)\right)\left(\frac{1}{q^{2}+N^{2}}-\frac{1}{q^{2}+(N+1)^{2}}\right) \\
= & \left(2 \frac{\ln (1+x)}{x} \frac{\varphi(q)}{q^{2}} P+O_{\varepsilon}\left(q^{-1 / 2+\varepsilon}+P q^{-3 / 2+\varepsilon}\right)\right) \frac{1}{q^{2}+P^{2}} \\
& +\sum_{N=1}^{P-1}\left(2 \frac{\ln (1+x)}{x} \frac{\varphi(q)}{q^{2}} N\right. \\
= & 2 \frac{\ln (1+x)}{x} \frac{\varphi(q)}{q^{2}} \sum_{N=1}^{P} \frac{1}{q^{2}+N^{2}}+O_{\varepsilon}\left(q^{-5 / 2+\varepsilon}\right) .
\end{aligned}
$$

Осталось только заметить, что

$$
\sum_{N=1}^{P} \frac{1}{q^{2}+N^{2}}=\int_{0}^{P} \frac{d \alpha}{q^{2}+\alpha^{2}}+O\left(\frac{1}{q^{2}}\right)=\frac{1}{q} \operatorname{arctg} \frac{P}{q}+O\left(\frac{1}{q^{2}}\right) .
$$

Лемма 5 полностью доказана.

\section{§4. Доказательство теоремы}

Пусть $R$ - произвольное натуральное число, большее единицы. Применяя лемму 2 , получим, что

$$
N_{x}^{*}(R)=\sum_{a^{2}+d^{2} \leqslant R^{2}}^{\text {НОД }(a, d)=1} s_{x}\left(\frac{a}{d}\right)=\sum_{1 \leqslant d<R} \Phi_{x}\left(d ; \sqrt{R^{2}-d^{2}}\right)+O\left(R^{2}\right) .
$$

Последняя сумма с погрешностью $O\left(R^{2}\right)$ есть число целочисленных пятерок $\left(m, m^{\prime}, n, n^{\prime}, \bar{n}\right)$, для которых

$$
m n^{\prime}+m^{\prime} n \leqslant R, \quad 1 \leqslant m^{\prime}<x m, \quad 1 \leqslant n<n^{\prime}, \quad \text { НОД }\left(m, m^{\prime}\right)=\operatorname{HОД}\left(n, n^{\prime}\right)=1,
$$

$$
|\bar{n}| \leqslant \sqrt{\left(R /\left(m n^{\prime}+m^{\prime} n\right)\right)^{2}-1} \cdot n^{\prime}, \quad \bar{n} n \equiv 1 \quad\left(\bmod n^{\prime}\right) .
$$


Положив для удобства $n^{\prime}=q$, отсюда получаем равенство

$$
N_{x}^{*}(R)=\sum_{1 \leqslant q<R} \sum_{\substack{1 \leqslant n<q \\|\bar{n}| \leqslant R}} \delta_{q}(n \bar{n}-1) G_{x}^{*}(q, n, \bar{n} ; R)+O\left(R^{2}\right),
$$

где $G_{x}^{*}(q, n, \bar{n} ; R)$ есть число пар натуральных $\left(m, m^{\prime}\right)$ с

$$
1 \leqslant m^{\prime} \leqslant x m, \quad m q+m^{\prime} n \leqslant \frac{q R}{\sqrt{q^{2}+(\bar{n})^{2}}}, \quad \text { НОД }\left(m, m^{\prime}\right)=1 .
$$

Величина $G_{x}(q, n, \bar{n} ; R)$ определяется теми же условиями за исключением условия взаимной простоты $m$ и $m^{\prime}$. Она совпадает с числом целых точек в треугольнике с вершинами

$$
(0,0), \quad\left(\frac{q R}{(q+x n) \sqrt{q^{2}+\bar{n}^{2}}}, \frac{x q R}{(q+x n) \sqrt{q^{2}+\bar{n}^{2}}}\right), \quad\left(\frac{R}{\sqrt{q^{2}+\bar{n}^{2}}}, 0\right) .
$$

Поэтому

$$
G_{x}(q, n, \bar{n} ; R)=(\text { площадь })+O(\text { периметр })=\frac{x}{2} \frac{q R^{2}}{(q+x n)\left(q^{2}+\bar{n}^{2}\right)}+O\left(\frac{R}{q+|\bar{n}|}\right) .
$$

Применяя вторую формулу обращения Мёбиуса, получим

Так как для $Q \geqslant 1$

$$
G_{x}^{*}(q, n, \bar{n} ; R)=\sum_{1 \leqslant t \leqslant R / q} \mu(t) G_{x}(q, n, \bar{n} ; R / t) .
$$

$$
\sum_{1 \leqslant t \leqslant Q} \frac{\mu(t)}{t^{2}}=\sum_{t=1}^{\infty} \frac{\mu(t)}{t^{2}}+O\left(Q^{-1}\right)=\left(\sum_{t=1}^{\infty} \frac{1}{t^{2}}\right)^{-1}+O\left(Q^{-1}\right)=\frac{6}{\pi^{2}}+O\left(Q^{-1}\right),
$$

TO

где

$$
G_{x}^{*}(q, n, \bar{n} ; R)=\frac{3 x}{\pi^{2}} \frac{q R^{2}}{(q+x n)\left(q^{2}+\bar{n}^{2}\right)}+W,
$$

$$
W \ll \sum_{1 \leqslant t \leqslant R / q} \frac{R}{t(q+|\bar{n}|)} \ll \frac{R}{q+|\bar{n}|} \ln \frac{R+q}{q} .
$$

В результате получаем, что

$$
N_{x}^{*}(R)=\frac{3 x}{\pi^{2}} R^{2} \sum_{1 \leqslant q<R} \sum_{\substack{1 \leqslant n<q \\|\bar{n}|<R}} \delta_{q}(n \bar{n}-1) \frac{q}{(q+x n)\left(q^{2}+\bar{n}^{2}\right)}+V,
$$

где

$$
V \ll \sum_{1 \leqslant q<R} \sum_{\substack{1 \leqslant n<q \\|\bar{n}|<R}} \delta_{q}(n \bar{n}-1) \frac{R}{q+|\bar{n}|} \ln \frac{R+q}{q}+O\left(R^{2}\right) .
$$

Так как

$$
\sum_{1 \leqslant n<q} \delta_{q}(n \bar{n}-1) \leqslant 1
$$


TO

$$
\begin{aligned}
V & \ll R \cdot \sum_{1 \leqslant q<R} \ln \left(1+\frac{R}{q}\right) \cdot \sum_{|\bar{n}|<R} \frac{1}{q+|\bar{n}|} \\
& \ll R \cdot \sum_{1 \leqslant q<R} \ln \left(1+\frac{R}{q}\right) \cdot \int_{0}^{R} \frac{d \alpha}{q+\alpha}=R \sum_{1 \leqslant q<R} \ln ^{2}\left(1+\frac{R}{q}\right) \\
& \ll R \int_{0}^{R} \ln ^{2}\left(1+\frac{R}{\alpha}\right) d \alpha=R^{2} \int_{1}^{\infty} \frac{\ln ^{2}(1+\beta)}{\beta^{2}} d \beta \ll R^{2} .
\end{aligned}
$$

Итак,

$$
N_{x}^{*}(R)=\frac{3 x}{\pi^{2}} R^{2} \sum_{1 \leqslant q<R} q \cdot U_{x}(R ; q)+O\left(R^{2}\right)
$$

где

$$
U_{x}(R ; q)=\sum_{\substack{1 \leqslant n<q \\|\bar{n}|<R}} \delta_{q}(n \bar{n}-1) \frac{1}{(q+x n)\left(q^{2}+\bar{n}^{2}\right)}
$$

Применяя лемму 5, получим

$$
N_{x}^{*}(R)=\frac{6}{\pi^{2}} \ln (1+x) R^{2} \sum_{1 \leqslant q<R} \frac{\varphi(q)}{q^{2}} \operatorname{arctg} \frac{R}{q}+O\left(R^{2}\right)
$$

Так как

$$
\operatorname{arctg} \frac{R}{q}=\frac{\pi}{2}+O\left(\frac{q}{R}\right)
$$

Tо

$$
N_{x}^{*}(R)=\frac{3}{\pi} \ln (1+x) R^{2} \sum_{1 \leqslant q<R} \frac{\varphi(q)}{q^{2}}+O\left(R^{2}\right) .
$$

Напомним, что (см. [7])

$$
\varphi(q)=q \sum_{t \backslash q} \frac{\mu(t)}{t}
$$

Поэтому

$$
\begin{aligned}
\sum_{1 \leqslant q<R} \frac{\varphi(q)}{q^{2}} & =\sum_{1 \leqslant q<R} \frac{1}{q} \sum_{t \backslash q} \frac{\mu(t)}{t}=\sum_{1 \leqslant q<R} \frac{\mu(t)}{t} \cdot \sum_{1 \leqslant t q^{\prime}<R} \frac{1}{t q^{\prime}} \\
& =\sum_{1 \leqslant t<R} \frac{\mu(t)}{t^{2}}\left(\sum_{1 \leqslant q^{\prime}<R / t} \frac{1}{q^{\prime}}\right)=\sum_{1 \leqslant t<R} \frac{\mu(t)}{t^{2}}\left(\ln \frac{R}{t}+O(1)\right) \\
& =\left(\sum_{t=1}^{\infty} \frac{\mu(t)}{t^{2}}\right) \ln R+O(1)=\frac{6}{\pi^{2}} \ln R+O(1) .
\end{aligned}
$$

Следовательно,

$$
N_{x}^{*}(R)=\frac{18}{\pi^{3}} \ln (1+x) R^{2} \ln R+O\left(R^{2}\right)
$$


и, окончательно,

$$
\begin{aligned}
N_{x}(R) & =\sum_{1 \leqslant t \leqslant R} N_{x}^{*}\left(\frac{R}{t}\right)=\sum_{1 \leqslant t \leqslant R}\left(\frac{18}{\pi^{3}} \ln (1+x)\left(\frac{R}{t}\right)^{2} \ln \frac{R}{t}+O\left(\frac{R^{2}}{t^{2}}\right)\right) \\
& =\frac{18}{\pi^{3}} \ln (1+x)\left(\sum_{1 \leqslant t \leqslant R} \frac{1}{t^{2}}\right) R^{2} \ln R+O\left(R^{2}\right)=\frac{3}{\pi} \ln (1+x) R^{2} \ln R+O\left(R^{2}\right) .
\end{aligned}
$$

Теорема полностью доказана.

\section{ЛитерАтУРА}

1. Арнолъд В. И. Цепные дроби. МЦНМО, М., 2000.

2. Задачи Арнольда. Фазис, М., 2000, задача 1993-11(С).

3. Авдеева М. О., Быковский B. А. Решение задачи Арнольда о статистиках ГауссаКузьмина. Препринт, Владивосток, Дальнаука, 2002.

4. Хинчин А. Я. Цепные дроби. Физматгиз, М., 1961.

5. Heilbronn $H$. On the average length of a class of finite continued fractions. In: Abh. Zahlentheorie Anal., VEB Deutsher Verlag der Wisenschaften, Berlin, Plenum Press, New York, 1968, pp. 89-96.

6. Сарнак П. Модулярные формы и их приложения. Фазис, М., 1998.

7. Виноградов И. М. Основы теории чисел. Наука, М., 1972.

Хабаровский государственный

Поступило в редакцию педагогический университет 16 февраля 2003 г.

Хабаровское отделение Института прикладной математики ДВО РАН email: mariya@iam.khv.ru 